be somewhat easier. Between November 1st and December 24th, ten abscesses developed in various parts of the body, most of which were aspirated or incised under novocain. On two occasions pis was sent for bacteriological examination and inoculation of a guinea-pig requested. "The first report was as follows: "Thick a guinea-pig requested. The first report was as follows: "Thick grumous from red blood corpuscles. Tubercle bacilli could not be apart from red blood corpuscles. Tubercle bacilli could not be " Microscopically it is essentially structureless, only a few celloutlines being seen. Tubercle bacilli could not be found either by direct films or by concentration methods. No other microorganisms wero found on microscopic examination and cultures remained sterile. Animal inoculation of the material for tubercle bacilli is being proceeded with."

During this time the patient's temperature and pulse remained about the same, his general condition seemed to be improving, but the signs in his chest were unaltered. The Wassermaun reaction was negative, as also were blood tests for typhoid and parat"yhoid infections. On December 12th his temperature began to subsiae, it being $98.4^{\circ} \mathrm{F}$. in the morning and $100^{\circ} \mathrm{F}$. in the evening; between December 18th and 22nd it remained between $97.2^{\circ} \mathrm{F}$. and $98.8^{\circ} \mathrm{F}$. On December $23 \mathrm{rd}$ it became subnormal, and remained so until his death six days later. The pulse rate, on the other hand, never dropped-below 100, ranging between 100 and 120 , with respirations constant at 24 .

Special features as regards the case from a differential diagnostic point of view were: (1) all the abscesses developed very quickly; (2) they caused great pain; (3) after the first had been incised the pus, which was quite innocuous, became so fetid that the patient had to be removed from the ward into a separate shelter; (4) the relief experienced after incision and aspiration was very considerable.

A. post-mortem examination was performed on December 30th by Dr. G. H. Pringle and myself, and the following observations were made.

There were adhesions on the left side between the left lung and chest wall, becoming denser towards the base. A large abscess was found in the base of the left lung, which showed collapse. Both lobes on the left side and the two upper lobes on the right contained numerous small nodules, which proved to be abscesses. The heart was small, and there was an ounce of greenish fluid in the pericardial sac. There was slight atheroma of the aorta, a little thickening around the pulmonary orifice, and a small abscess in the wall of the left ventricle. Other abscesses were found in the following organs: two in the liver which was congested and fibrous; one in the left kidney, round which were adhesions; a perinephric abscess, which communicated which were adhesions; a perinephric abscess, which communicated
with the one in the lower part of the left lung through an opening in the diaphragm (the right kidney was normal); and one in the spleen, which was slightly enlarged and friable. There were a - few small flattened greyish nodules in the mesentery, but no peritoneal adhesions.

Portions of the abscess tissues from the liver, lungs, and heart were examined histologically, and the organism responsible for the condition was found to be the actinomyces. The tissue was remarkable in showing a large number of granules, which is rare in human actinomycosis. The guinea-pigs inoculated showed no signs of tuberculous infection, and the pus was presumably, therefore, free from tubercle bacilli.

My thanks are due to Dr. H. Hyslop Thomson, county medical officer of health, for permission to publish this account, and for his assistance, also to Dr. G. H. Pringle for his help in the investigation.

\section{AN ADJUSTABLE BOARD-BED.}

LEONARD HEARN, M.B., B.S.DURH.,

LATE RESIDENT MEDICAL OFFICER, HULL TUBERCULOSIS SANATORIUM, COTTINGHAM, E. YORKS.

Túbercurous disease of the hip is unfortunately only too often met with for the first time when the condition is well established and the joint is more or less fixed, with the linib in a bad position of adduction, inward rotation and flexion, and usually a certain amount of shortening. The bed splint to be described has been used with excellent results; it is designed to correct tho defornity very gradually and obtain the desired position, with a quiescent condition, before the limb is put up in a plaster splint.

All tuberculous lesions improve as the general condition of the patient grows better, and these cases should be placed on the open verandah day and night in all seasons. 'This bed splint enables the patients to be treated under open-air conditions; they are really only confined to bec like pyrexial pulmonary cases, and enjoy a maximum amount of comfort, thus benefiting both morally and physically. Often in cases of tuberculous disease of the hip-joint the limb is fixed in plaster, or in a Thomas's hip splint, in a more or less good position, but this unfortunately may entail a compensatory lordosis of the spine, with subsequent permanent deformity.

This apparatus is essentially a " board-bed," hinged in the middle so that the lower half supporting the legs may be raised or lowered at will by means of a support on a ratchet similar to the method of fixing an ordinary deck chair. Six boards form a box frame for retaining the patient in position, and these swivel from the centre line

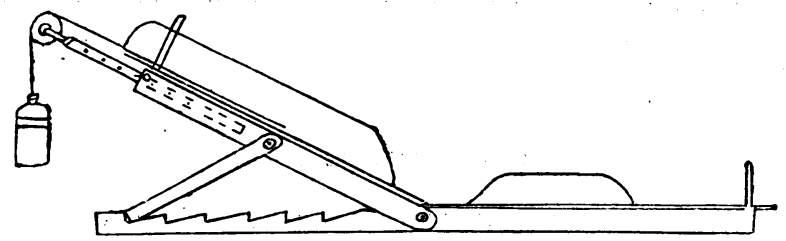

of the bed; they are kept. in position by means of pegs in rows of holes at the foot and head. The lower end of the frame can be extended to fit any length of patient, and, the boards being $3 \frac{1}{2}$ to 4 inches high, cradles are unnecessary. Pulleys adjusted by means of thumbscrews carry the extension weights, and move to any position on the lower edge of the franie.

At the commencement of treatment the spine is placed in its natural position, with the limbs raised and with a suitable extension weight applied, the affected joint is gradually abducted, and the leg is lowered as required, the position of the spine not being allowed to vary, so that lordosis is never preseitit.

The case shown is a typical tuberculous arthritis of the left hip; this joint was held in a bad position of flexion and adduction, with two inches of shortening. There were present also all the symptoms of active disease, such as pain, heat, and pyrexia.

The first photograph demonstrates the amount of compensatory lordosis which was present when the affected

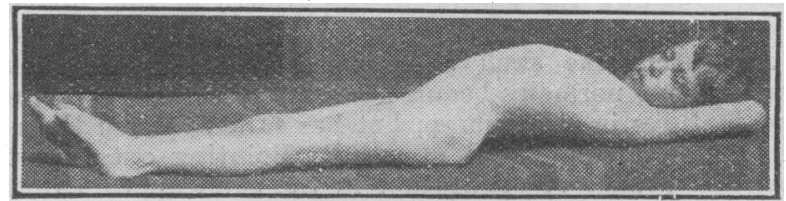

ley was kept flat to correspond with the sound leg; this is the position in which so many limbs, after abduction and external rotation, are finally fixed in a plaster-of-Paris or

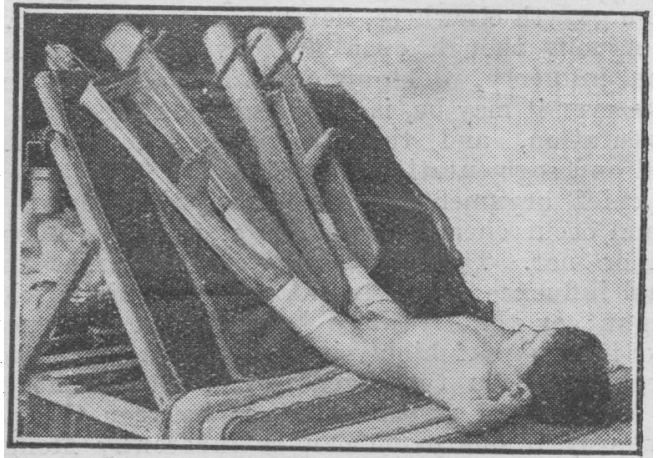

Thomas's splint. The second photograph shows the patient in position at the commencement of treatment, with the spino in normal position. In the third the corrected limb

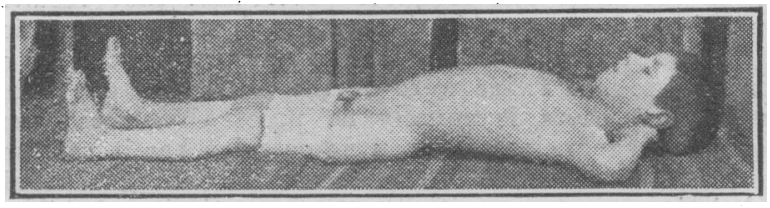

is seen after use of the board-bed and ready to be put up in plaster-of-Paris, after having been submitted to very gradual lowering and extension, dependent on the position of the spine. 
This patient was never off an open verandah. His general condition is excellent in every way; there is now no pain or tenderness, and the pulse and temperature are quite normal.

The apparatus is simple to use; it can be quickly adjusted in any direction by the medical officer from time to time, and it renders nursing easy. Thus a bed-pan is used by nerely hooking up the extension reights and sliding the patient up the bed; he fits back into position when the pan is removed, and the weights again hang free. The splint will lie on any ordinary bed and fit any patient, child or adult. Cradles, which cause draught and discomfort, are rendered unnecessary.

\section{Altemuranda: MEDICAL, SURGICAL, OBSTETRICAL.}

\section{FRACTURED BASE OF THE SKULL FOLLOWED} BY ACUTE MASTOID DISEASE: RECOVERY.

AcUTE mastoid disease is an unusual sequel of fractured base, and the following details of such a case seem to be worthy of record, especially since almost complete recovery followed operative treatment.

While hunting on February 1st, 1926, a lady was thrown from her horse, which rolled over her. She was un was thrown from picked up, and was bleeding from both ears and from the nose. poth clavicles were broken, and there were injuries to the nose. Both clavicles were broken, and there were injuries to some ribs. She was completely uncouscious for twelve hours, and only partially
conscious for the next four days. It was noticed that she had right facial paraiysis, and on recovering consciousness more com pletely it was found that she had paralysis of the right sixth nerve, causing double vision.

The external auditory canals were kept clean by swabbing with 1 in 20 carbolic solution. On the eighth day there was earache on the right side; a discharge appeared from both ears on the tenth day, and her temperature rose to $100^{\circ} \mathrm{F}$. on ears on the day she complained of earache on the left side. There was considerable swelling around the upper part of the right mastoid, just below the zygoma. This had appeared very shortly after the accident. There was a free discharge of pus from this ear, and when the canal was cleaned out it was found to be torn across close to the membrane, which could not be seen properly owing to narrowing of the canal. The left canal contained some dried blood and discharge, but no perforation could be seen in the membrane, which was not reddened. Both mastoids were very membrane, which was not reddened. Both mastoids were very tender to pressure, and this had always been the case since she
recovered consciousness. Air conduction was diminished but bone recovered consciousness. Air conduction was diminished, but bone
conduction was normal. The temperature was $100.6^{\circ} \mathrm{F}$., and the pulse 92. She answered questions intelligently, but when not spoken to lay in a drowsy condition with her eyes closed. The following day the pulse and ternperature wcre normal, and remained so for thirty-six hours, when the temperature again rose to $100^{\circ} \mathrm{F}$., the pulse being 88 . On the sixteenth day she had "neuralgic", pain in her right ear and mastoid, and there was some increase of the swelling in this region, the ear being pushed forward; the temperature was $101.6^{\circ} \mathrm{F}$, and the pulse 100 . The left ear was again discharging slightly, and it was obvious that the right mastoid was infected.

First opcration.-The usual incision was made, and a fracture could be felt; on retracting the tissues it was seen to run upwards and backwards over the mastoid from the lower part of the bony meatus, and there was some saping between the edges. A small three-sided trocar was used to make a hole in the bone; this was replaced by larger-sized trocars, and by gentle use of burrs and forceps the hole was enlarged. Some pus was found in the cells just over the antrum, and on opening the latter there was a gush. of pus. The surrounding cells were removed, but no attempt was made at a formal operation. The middle ear was washed out through the auditus, and the cavity in the bone, after being mopped out with spirit, was bipped and lightly packed with bipped gauze. The perforation in the left membrane was entarged. The time of operation was 11 p.m.

The next morning at 8 o'clock the temperature was $98.4^{\circ} \mathrm{F}$. the temperature had risen to $103^{\circ} \mathrm{F}$. pulse 132; the breathing was sighing, and she appeared very il ndeed. There were no meningeal or labyrinthine symptoms, but the question of a commencing lateral sinus infection or a perisinous abscess had to be considered, as had also a deep infection of the tissues in the swollen area or in the line of the fracture. By the evening the temperature had dropped to $100.6^{\circ} \mathrm{F}$., pulse remained below $100^{\circ} \mathrm{F}$. till the twentieth day when it again rose to $101.4^{\circ} \mathrm{F}$. at 8 a.m., and $101.4^{\circ} \mathrm{F}$. at 4 p.m. the next day. The swelling in the temporal and zygomatic region became more marked, the right eyelid was swollen, and the eye was more closed; some oedema and reddening over the forehead appeared, extending to the left side.

Second Opcration:-An incision was made in the temporal region, and the temporal, zygomatic, and spheno-maxillary regions were explored with a very large-bore needle, but no pus was found. A. further free incision in the temporal region was made and fomentations were applied. The next day the temperature again shot up to $103^{\circ} \mathrm{F}$, but dropped to $98^{\circ} \mathrm{F}$. by $8 \mathrm{p.m}$.; after this convalescence was rapid and uneventful.

The facial paralysis had almost entirely clcared up by the beginning of May, when she was able to take walks of iwo to four miles, but the diplopia persisted for about another month. Some granulations had to be curetted out of the right canal. The discharge soon cleared up. As soon as the membranes were soundly healed gentle politzerization was used, and this was later supplemented by massage. From time to time all treatment was stopped for a week to ten days.

The hearing in both ears is now practically normal; there is no narrowing of the right external auditory canal. The patient is again leading her normal life, and has resumed hunting. Headaches, or other sequelae, are absent.

W. J. Harrison, M.B., B.S.

Surgeon, Ear, Nose, and Throat Hospital, Newcastle-on-Tyne.

\section{PNEUMONIA WITH ACUTE ABDOMINAL SYMPTOMS} AND MULTIPLE ABSCESSIS.

The simulation by pneumonia of an acute abdominal condition is not very uncommon, but the following case presents some unusual features and seems, therefore, to be worthy of record.

A young lascar was admitted to the British Cottage Hospital at Algiers, on February 16th, complaining of anorexia and great pain below the liver running down the right side of the abdomen. On palpation muscular contraction prevented exploration of the subjacent viscera, but no tympanites was present and the abdominal respiration was unimpeded. The temperature was $101^{\circ}$. The bowels were loose, but nothing abnormal was found in the faeces; it was decided that there was no necessity for immediate intervention.

$\mathrm{He}$ began to cough considerably two days after admission, and He began to cough considerably two days after admission, and examination showed dullness oyer the upper lobe of the right lung extending into the axilla. The sputum was not particularly viscid and did not present the characteristic colour of pneumonic
expectoration. Puncture of the pleura over the dull area brought away only a few drops of blood-stained fluid rich in pneumococci. At the back respiration at the upper part of the lung was blowing and there was pectoriloquy. The crisis occurred on February 25th, when the temperature returned to normal and did not subsequently rise.

The patient improved and began to take food readily, but he complained of pain in the back of the neck on movement in any direction. Nothing abnormal could be made out in that situation by palpation, but there may have been pneumococcal arthritis; Kernig's sign was absent. Then swellings appeared on the arms-one on the outer aspect of the right forearm and two on the outer aspect of the left arm, the uppermost just below the deltoid. The swellings ranged in size from a filbert to a wainut, and fluctuated freely. On being opened they gave exit to creany pus containing a pure culture of pneumococci. The patient was given three intravenous injections of antipnetimococcal vaccine; these were not followed placed in a separate ward. He ultimately made a good recovery, placed in a separate ward. He ultimately made a good recovery, the abdominal symptoms having cleared up spontaneously in the
course of four or five days; the stiffness of the neck subsided gradually.

Judging from the literature of the subject the prognosis in cases of pneumococcaemia before the introduction of raccines was extremely grave, but this is my second case in which the use of an antipneumococcal vaccine appears to have exerted a favourable influence. I reported a case of pneumococeal polyarthritis in the British Medical Journal of September 13th, 1924 (p. 455).

The interesting feature in the present instance is the patient's admission as an acute abdominal case. The fact that pneumonia may simulate appendicitis has long been known, and this is particularly the case in infantile pneumonia. Daussy, in 1913, collected notes of a number of such cases for his Paris thesis. He points out that while some patients treated for pneumonia were found after death to have had appendicitis, on other occasions patients operated upon for appendicitis were found after death to have had pneumonia. He concluded that pneumonia, especially in children, might be accompanied by an abdominal syndrome resembling that of appendicitis. This is not due to an abnormal localization of the pneumococcus; it is quite possible, of course, for the two affections to coexist, and it is obvious that an attack of appendicitis may predispose to an attack of pneumonia. He divides these cases into two main groups-namely, (1) those in which the pneumonia soon dominates the scene while the abdominal symptoms spontancously clear up, and (2) those in which the appendicitis becomes and remains the principal feature, the pneumonia only being recognized later on. The cases belonging to the first group are usually mild, but the prognosis is not so good in the second. The moral to be drawn from his observations is that in 